\section{Nasensprays: Kosten in Theorie und Praxis}

$\mathrm{N}$ asale Glukokortikoide zählen zu den Standardtherapeutika in der Allergologie. Wie in jeder Arzneimittelgruppe gibt es aber auch hier Unterschiede im Wirksamkeits- und Sicherheitsprofil. Über ein gutes Nutzen-Risiko-Profil verfügt das MometasonfuroatNasenspray Nasonex ${ }^{\circledR}$. Seine systemische Bioverfügbarkeit liegt unter $0,1 \%$ und ist damit praktisch vernachlässigbar - oder wie es Prof. Dr. Ludger Klimek vom Wiesbadener Zentrum für Rhinologie und Allergologie in einem Interview formulierte: „Mometason entfaltet dort seine Wirkung, wo sie gebraucht wird, nämlich an der Nasenschleimhaut." Verschiedene klinische Studien belegen eine ausgeprägte antientzündliche Wirkung von Mometason. Diese macht sich sowohl in einer guten Compliance wie auch in geringeren Verbrauchsmengen im Vergleich zu anderen Glukokortikoid -Sprays bemerkbar. „Bei einigen älteren Präparaten benötige ich für einen therapeutischen Erfolg vier bis acht Hübe, während ich bei Mometason häufig mit der halben Hubzahl auskomme", berichtete Klimek.

Dieses Beispiel zeigt, dass die im Arzneiverordnungs-Report herangezogene definierte Tagesdosis („defined daily dose“, DDD) von $0,2 \mathrm{mg}$ für nasale Glukokortikoide nicht immer der Praxisrealität entspricht. Ursprünglich wurden die DDDs von der WHO lediglich für empirische Vergleiche des Verordnungsverhaltens von Ärzten auf Länderebene entwickelt. Die Verwen-

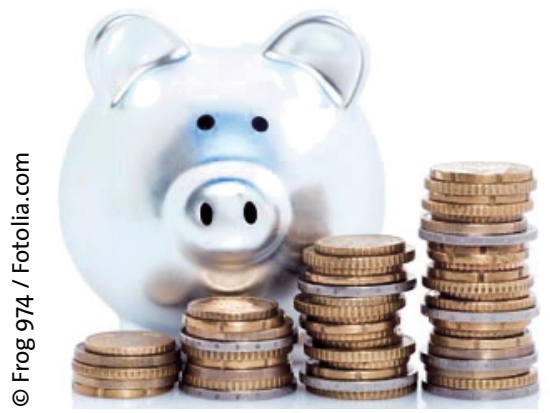

Bares Geld wird durch die Wahl eines effektiven Glukokortikoidsprays gespart bei der Kostenberechnung zählt nämlich nicht die definierte Tagesdosis, sondern der tatsächliche Verbrauch. dung der DDDs für Wirtschaftlichkeitsvergleiche lehnt die WHO sogar explizit ab. Eine kürzlich publizierte Pharmaökonomiestudie bei niedergelassenen Ärzten (Schöffski O et al. Gesundh ökon Qual manag 2010; 15: 192-8) arbeitet daher mit der im Praxisalltag tatsächlich verordneten Dosis („prescribed daily dose“, PDD). Klimek unterstrich: „Das ist ein realistisches $\mathrm{Maß}$ und dieses sagt dann auch etwas darüber aus, was man tatsächlich wirtschaftlich aufwenden muss, um einen definierten therapeutischen Erfolg zu erreichen. Und das ist dann auch für verschiedene Präparate vergleichbar."

Die Pharmaökonomiestudie zeigte, dass ein zunächst aufgrund von DDDBerechnungen günstiger erscheinendes generisches Budesonid-Präparat in der Praxis weniger wirtschaftlich ist als die Mometason-Alternative. Ausgewertet wurden die Daten von 14.767 Patienten mit Nasonex ${ }^{\circledR}$-Verordnungen und 2.489 Patienten mit Budesonidhaltigem Nasenspray (50 $\mu \mathrm{g}$ Wirkstoff) aus allgemeinmedizinischen Praxen sowie von 8.817 mit Mometason und 748 mit Budesonid behandelten Patienten aus HNO-Praxen. Der Erhebungszeitraum war von Oktober 2004 bis September 2008.

Unter diesen Realbedingungen unterschieden sich die beiden Glukokortikoidpräparate trotz gleicher DDDAngaben im Arzneiverordungs-Report beim tatsächlichen Verbrauch deutlich zugunsten von Mometason. Unabhängig von der Diagnose ergab sich für Mometason nach 730 Tagen ein Minderverbrauch pro Jahr von 227 Hüben beim HNO-Arzt und von 132 Hüben beim Allgemeinarzt. Auch waren Folgeverordnungen und Präparatewechsel unter Mometason seltener als unter Budesonid. „Die Verordnung von Mometason ist meines Erachtens nach wirtschaftlich und preisbewusst. Eventuell können durch die Verordnung von Mometason sogar Kosten im Sinne der Solidargemeinschaft gespart werden", so das Fazit von Klimek.

red

Nach Informationen von MSD, Haar

\section{Handelszulassung in Indien}

Der Import, Verkauf und Vertrieb der allergologischen Diagnostik-Produktpalette des schwedischen Unternehmens Phadia - zu der die Allergene der ImmunoCAPSerie und das System zur Messung von Autoantikörpern EliA gehören - sind im Dezember letzten Jahres von den indischen Behörden genehmigt worden. Der indische Markt ist für Phadia strategisch bedeutend: Mit einer geschätzten Prävalenz von Allergien von über $30 \%$ und einer Gesamtbevölkerung von 1,2 Milliarden Menschen liegt in diesem Land ein erhebliches Wachstumspotenzial für den Labormarkt. Schon im April 2010 eröffnete Phadia eine eigene Niederlassung in Indien und arbeitet dort inzwischen mit mehreren der großen Handelslabore und privaten Krankenhausketten zusammen.

Nach Informationen von Phadia, Uppsala, Schweden

Pneumologische Ausflüge

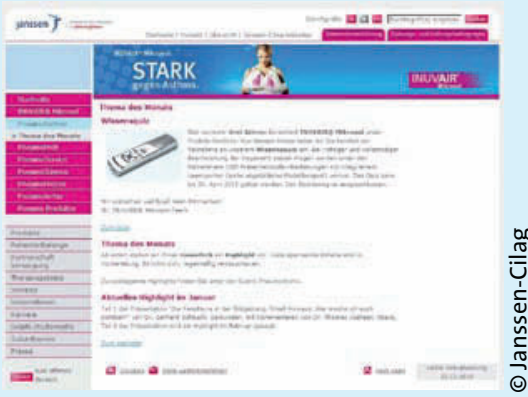

Seit Ende 2010 tritt die Website www.inuvairmikrosol.de in einem neuen und anwenderfreundlichen Outfit auf. Als Highlight wird unter der Rubrik „PneumoPartner" jeden Monat ein neues pneumologisches Thema vorgestellt. Den Anfang machte im Dezember eine von Prof. Dr. J. Christian Virchow aus Rostock kommentierte Zusammenfassung des Übersichtsartikels „pharmacogenomic approaches to asthma treatment". Interessante Einblicke in die Welt der kleinen Atemwege verspricht im Januar die Präsentation des Physikers Dr. Gerhard Scheuch aus Gemünden mit dem Thema: „Die Peripherie in der Bildgebung". Begleitet wird die Reise ins Innere des Menschen durch den Pneumologen Dr. Thomas Voshaar aus Moers. Ärzte und Apotheker, die sich im Bereich Pneumologie weiterbilden wollen, sollten regelmäßig vorbeischauen. red

Nach Informationen von Janssen-Cilag, Neuss 